\title{
A simple novel technique to induce short-lasting local brain ischaemia in the rat
}

Knapp L. ', Gellért L. . , Herédi J. ', Kocsis K. ', Oláh G. ', Fuzik J. , Kis Zs.., Vécsei L. ${ }^{2.3}$ and Toldi J., ${ }^{1,3}$, Farkas T. ${ }^{1}$

1Department of Physiology, Anatomy and Neuroscience, University of Szeged, Közép fasor 52, H6726 Szeged, Hungary

2Department of Neurology, University of Szeged, POB 427, H6701 Szeged, Hungary ${ }_{3}$ Neurology Research Group of the Hungarian Academy of Sciences and University of Szeged

Key words: brain ischaemia, middle cerebral artery occlusion (MCAO), TIA, stroke, somatosensory evoked potentials, Fluoro Jade C, astrocytes, microglia

Running title: Ischaemia-reperfusion by lifting MCA

Corresponding author:

József Toldi, Ph.D., D.Sc.

Department of Physiology, Anatomy and

Neuroscience, University of Szeged,

POB 533, H6701

Szeged, Hungary

email: toldi@bio.uszeged.hu

Tel.: +3662544153

Fax.: +3662544291 


\section{Abstract}

Aims: Brain ischaemia models are essential to study the pathomechanisms of stroke. Our aim was to investigate the reliability and reproducibility of our novel focal ischaemia-reperfusion model.

Methods: To induce a cortical transient ischaemic attack, we lifted the distal middle cerebral artery (MCA) with a special hook. The early changes after 2x15-min occlusion were observed in the somatosensory evoked responses (SERs). The histological responses to 2x15-min MCA occlusion and to 30,45 or 60 -min ischaemia were examined after a 1-day survival period by 2,3,5-triphenyltetrazolium chloride (TTC) and Fluoro Jade C (FJC) staining. Another group, with 30-min ischaemia, was analysed histologically by FJC, S100 and CD11b labelling after a 5-day survival period.

Results: The amplitudes of the SERs decreased immediately at the beginning of the ischaemic period, and remained at a reduced level during the ischaemia. Reperfusion resulted in increasing SER amplitudes, but they never regained the control level. The short-lasting ischaemia did not lead to brain infarction when evaluated with TTC, but intense labelling was found with FJC. The 30-min ischaemia did not result in FJC labelling after 1 day, but marked labelling was observed after 5 days with FJC, S100 and CD11b in the cortical area supplied by the MCA.

Conclusions: We present here a novel, readily reproducible method to induce focal brain ischaemia. The ischaemia-reperfusion results in noteworthy changes in the SERs and the appearance of conventional tissue damage markers. This method involves possibilities for precise blood flow regulation, and the setting of the required level of perfusion. 


\section{Introduction}

During the past 30-35 years, there have been significant developments as concerns animal models of cerebral ischaemia designed to shed light on the pathomechanism leading to tissue damage, with the aim of the discovery of new therapies for stroke [1,2]. For a considerable time, middle cerebral artery occlusion (MCAO) has been one of the models widely used to induce reversible brain ischaemia [3-6]. In most cases, a variety of intraluminar nylon filaments have been utilized in rat MCAO models. However, the extent of the lesion and its reproducibility tend to vary from laboratory to laboratory. The method itself is rather complex, but the properties of nylon filaments are also responsible in part for these variations [7]. Moreover, when short-lasting (15-30-min) ischaemia is required, e.g. in studies of the effects of transient ischaemic attacks (TIAs), there is no major tissue damage and no infarcts, and it is therefore particularly difficult to achieve and check reproducibility. However, there is no animal model that can be used to induce focal, well-regulated repetitive ischaemiareperfusion. In this paper, we present a simple new method for the induction of transient focal brain ischaemia.

\section{Materials and methods}

\section{Animals and MCAO}

Male adult Wistar (Charles River) rats weighing 250-300 g $(\mathrm{N}=34)$ were used in the experiments. The animals were given free access to food and water prior to surgery. All procedures were approved by the Animal Care Committee of the University and were conducted in accordance with the Directives of the European Union. Experiments were carried out under Nembutal anaesthesia. Body temperature was maintained at $37 \pm 0.5^{\circ} \mathrm{C}$ 
through use of a self-regulating heating pad (Supertech TMP5a) and a rectal probe. After removal of the masticatory muscle, the head of the animals was fixed in a stereotaxic head holder. The surface of the left side of the temporal skull was cleaned and the brain was exposed with a high-speed microdrill. Saline was applied to the area throughout the procedure in order to prevent heat injury and to maintain the area in a hydrated state. The exposed cortical surface involved the trunk and main branches of the MCA and the barrel field in the primary somatosensory cortex (Fig. 1). To induce ischaemia at a point where its diameter was $300 \mu \mathrm{m}$, the MCA was carefully lifted through $1200 \mu \mathrm{m}$ with a Fisher microsurgery hook

with the aid of a micromanipulator. The ischaemic duration was adjusted to $2 \times 15$ (interrupted by a 30-min reperfusion), 30, 45 or $60 \min (\mathrm{N}=5,3,3$ and 3$)$. To terminate the occlusion, the hook was carefully removed, and restoration of the blood flow was confirmed under an operating microscope. Finally, the dura and the temporal muscle were replaced, the skin was closed with a silk suture and the wound was cleaned with iodine solution. The procedure was quick and secure; the survival rate was 95\% (34/36 animals).

\section{Electrophysiology}

Somatosensory evoked responses (SERs) induced as described previously [8] were recorded in 8 animals before, during and after the ischaemic period (120 min). Briefly, the trigeminal nerve was stimulated by electrical stimulation of the whisker pad $(4 \mathrm{~V}, 0.2 \mathrm{~ms}, 0.1 \mathrm{~Hz})$ through a bipolar needle electrode $[8,9]$. The recordings were made on the surface of the dura with the aid of a silver electrode. The punctum maximum of the SERs was identified; it was generally localized $3.5 \mathrm{~mm}$ behind the bregma and $5 \mathrm{~mm}$ laterally (Fig. 1). The amplified responses were processed and averaged with Experimetria Intrasys software (Experimetria Ltd., Budapest, Hungary). The 30-min control period was followed by 2 x 15-min ischaemic episodes interrupted by a 30-min reperfusion. 


\section{Histology}

For histological assessment after a 1 or 5-day survival period following surgery, the animals $(\mathrm{N}=26)$ received an overdose of urethane, and were perfused transcardially with $0.1 \mathrm{M}$ icecold phosphate-buffered saline (PBS, $\mathrm{pH} 7.4$ ), followed by $4 \%$ buffered paraformaldehyde. The brains were removed, and postfixed overnight in paraformaldehyde at $4{ }^{\circ} \mathrm{C}$. Coronal sections $(20 \mu \mathrm{m})$ were obtained with a vibratome (Leica VT1000 S). The early changes in neural viability induced by the ischaemia and reperfusion were visualized by means of 2,3,5triphenyltetrazolium chloride (TTC, 1.5\%) and Fluoro Jade C (FJC) staining in 14 animals. After 5 days, the delayed consequences of the 30-min ischaemic attack were also demonstrated with FJC, and were assessed with the conventional free-floating immunohistochemistry of reactive astrocytes (rabbit polyclonal antiS100, dilution 1:2000; DAKO) and activated microglia (mouse monoclonal anti-rat CD11b, clone OX-42, dilution 1:1000; Millipore) in 12 animals. Three adjacent slices were obtained at $500 \mu \mathrm{m}$ from bregma 1 to 4.5. Briefly, the slices were washed in PBS, then incubated in a blocking solution containing $10 \%$ normal donkey serum (NDS), and next incubated in primary antibody overnight at $4{ }^{\circ} \mathrm{C}$ and in secondary antibody for $2 \mathrm{~h}$ at room temperature. The antibodies were diluted in a solution containing $0.1 \%$ PBS, $0.4 \%$ Triton X100, $2 \%$ NDS and $0.01 \%$ sodium azide. The slices were coverslipped with Fluoromount G. Fluorescence photomicrographs were obtained with an Olympus BX51 microscope fitted with a DP70 digital imaging system. Solvents were obtained from Sigma-Aldrich Co.

\section{Statistical analysis}


Statistical analysis of electrophysiological data was performed with the General Linear Model/Repeated Measures (PASW Statistics 18 data analysis package, SPSS Inc., Chicago, IL, USA).

\section{Results}

\section{Electrophysiology}

The amplitudes of the SERs decreased immediately at the beginning of the ischaemic period, and remained at $8-12 \%$ of the control level. Reperfusion resulted in a gradual increase in the amplitudes of the SERs, though the amplitudes never regained the control level, but only around $60 \%$ of it. This phenomenon could be reproduced with a high level of significance in the same animal (Fig. 2).

\section{Histology}

The short-lasting 2 x 15-min ischaemic period did not induce a degree of tissue damage after a survival time of 1 day that was sufficiently severe to be detected with TTC staining at the various stereotaxic coordinates (Fig. 3A). After a 30, 45 or 60 -min ischaemic period, no FJC staining positivity was observed. However, as a result of the 2 x 15-min MCAO, well-outlined FJC-positive cells were seen throughout the ipsilateral somatosensory cortices supplied by the MCA. Staining was pronounced in the cell membrane and the cytoplasm (Fig. 3C). FJCpositive cells could readily be determined as the FJC did not penetrate into the damaged brain parenchyma during the 1-day survival period. No labelling occurred on the contralateral side (Fig. 3B). In another series of experiments with a 5-day survival period, we examined the changes with FJC, and investigated whether conventional glial responses are observed as a consequence of 30-min MCAO. Positive labelling was observed in half of the animals in all 
three histological processes. The damaged cells labelled by FJC were found in an extensive area of the ipsilateral cortex (Fig. 4B). S100 immunohistochemistry revealed an increased number of S100-positive astrocytes and a typical change in astrocyte phenotype in response to the 30-min MCAO (Fig. 4D). The ipsilateral cortex was characterized by hypertrophic astrocytes with prominent, thick processes and small vacuoles in the cell bodies (Fig. 4D, insert). The number and phenotype of the S100-positive astrocytes on the contralateral side were normal (Fig. 4C). Immunostaining of the activated microglia also revealed the activated phenotype of these cells. On the contralateral side, the microglia stained faintly, revealing a normal, resting, ramified morphology (Fig. 4E). As a result of the 30-min MCAO, the microglia ipsilaterally displayed an activated phenotype with enlarged somata and the loss of secondary and tertiary branching, which is typical in pathological conditions (Fig. 4F, and insert).

\section{Discussion}

We have presented here a novel, readily reproducible method for the induction of focal transient ischaemia in the rat cortex. Stroke, as the second leading cause of death worldwide, is studied intensively. The use of animal models in recent years has improved our understanding of the pathomechanism of this disease. The animals most widely used in such models are rats and mice [2]. In rats, the most frequent methods are based on mechanical occlusion of the proximal MCA [10]. In models which involve distal occlusion, as in our present model, the damage is restricted to the cerebral cortex, and the models are highly reproducible [11]. Reproducibility is a particularly difficult problem when the aim is to study the effects of short-lasting ischaemic attacks, e.g. TIAs. In these cases, there is no serious tissue damage, no infarct, and therefore no infarct volume which could be studied by TTC 
labelling. In transient ischaemia, the final injury is a result of ischaemic and reperfusion damage. The reperfusion itself provides substrates of enzymatic oxidation reactions and proinflammatory mediators $[12,13]$. Although biochemical and molecular biological studies are needed to identify in detail the activated pathways, the method that we have reported here for the induction of fine changes in the cortex with TIAs is simple and well reproducible with an extremely high survival rate. A short-lasting period of occlusion, achieved by lifting of the MCA where its diameter is $300 \mu \mathrm{m}$, resulted in a characteristic and significantly reproducible decrease in the amplitudes of the SERs. The histological study on brain slices taken from a definitive distance from the MCAO after 2 x 15-min ischaemia indicated FJC labelling. Although the emergence of FJC staining after a relatively short survival period ( 1 day in our experiment) after MCAO has not been described in the literature, in our experimental model and paradigm well-outlined FJC-positive cells were found throughout the ipsilateral somatosensory cortices. In contrast with the ipsilateral cortex, no staining at all was observed in the contralateral hemisphere. The mechanisms of staining with FJ dyes are not clearly understood [14], but our results lead us to suppose that the repeated ischaemia-reperfusion induced consecutive processes that resulted in molecules which bind FJC. The phenomenon that $2 \times 15$-min ischaemia did, whereas 30,45 or 60 -min ischaemia did not induce FJC staining after a 1-day survival may indicate different intracellular signalling processes. These processes may result in molecules that bind FJC, but this does not necessarily mean a greater extent of damage in the brain tissue.

In the case of a 5-day survival period, the effect of slight (30-min) ischaemia could be detected with FJC. Astrocytes and microglial cells suffer changes after a transient ischaemic insult $[11,15,16]$; indeed, with our model we also observed characteristic changes in the glia, in the injured cortical areas, but not in the contralateral cortex.

Pathophysiological processes of the ischaemic condition itself, induced for example by a TIA 
or short-term hypoperfusion, overlap with the excitotoxic processes following ischaemic periods (especially the reperfusion). Although a spontaneous reperfusion occurs in approximately $21 \%$ of cases of human stroke [17, 18], TIA allowed us to investigate endogenous survival mechanisms. The experimental study of focal TIAs is therefore reasonable. Our new method offers an easy way to study the effects of TIAs with precise regulation of the blood flow, and to set the required level of perfusion.

To summarize, we have achieved a simple and highly reproducible model for the induction of short-lasting, focal transient cortical ischaemia in the rat by lifting up the distal MCA.

\section{Acknowledgements:}

Knapp L. conceived the idea, designed the surgical technique and performed the measurement of electrophysiology. Toldi J. supervised the investigations and wrote the paper. Farkas T. and Kis Zs. conducted the experiments. Gellért L. and Herédi J. made the histological examinations. Kocsis K., Oláh G. and Fuzik J. analyzed the data and edited the figures. Vécsei L. was the theoretical advisor. All authors approved the final version.

The authors thank Gabriella Mészáros for her technical support, animal care, handling and breeding. We are grateful to David Durham for linguistic correction of the manuscript. This study was financially supported by grants from TÁMOP 4.2.2-A-11/KONV-2012-0052 and OTKA K105077 and the Hungarian Academy of Sciences. This research was realized in the frames of TÁMOP 4.2.4. A/2-11-1-2012-0001 „National Excellence Program - Elaborating and operating an inland student and researcher personal support system". The project was subsidized by the European Union and co-financed by the European Social Fund.

Author considerations

K.L., K.K. and F.J. hold predoctoral grants from the Hungarian Ministry of Education. F. T. 
was a Bolyai Fellow of the Hungarian Academy of Sciences.

\section{References}

1 Bacigaluppi M, Comi G, Hermann DM. Animal models of ischemic stroke. Part two: modeling cerebral ischemia. The open neurology journal 2010; 4: 34-8

2 Casals JB, Pieri NC, Feitosa ML, Ercolin AC, Roballo KC, Barreto RS, Bressan FF, Martins DS, Miglino MA, Ambrosio CE. The use of animal models for stroke research: a review. Comparative medicine 2011; 61: 305-13

3 Relton JK, Beckey VE, Hanson WL, Whalley ET. CP-0597, a selective bradykinin B2 receptor antagonist, inhibits brain injury in a rat model of reversible middle cerebral artery occlusion. Stroke; a journal of cerebral circulation 1997; 28: 1430-6

4 Seiffge DJ, Lapina NE, Tsagogiorgas C, Theisinger B, Henning RH, Schilling L. Improvement of oxygen supply by an artificial carrier in combination with normobaric oxygenation decreases the volume of tissue hypoxia and tissue damage from transient focal cerebral ischemia. Experimental neurology 2012; 237: 18-25

5 Sopala M, Danysz W, Quack G. Neuroprotective effects of NS-7, voltage-gated Na+/Ca2+ channel blocker in a rodent model of transient focal ischaemia. Neurotoxicity research 2002; 4: $655-61$

6 Vaibhav K, Shrivastava P, Javed H, Khan A, Ahmed ME, Tabassum R, Khan MM, Khuwaja G, Islam F, Siddiqui MS, Safhi MM, Islam F. Piperine suppresses cerebral ischemia-reperfusion-induced inflammation through the repression of COX-2, NOS-2, and NF-kappaB in middle cerebral artery occlusion rat model. Molecular and cellular biochemistry 2012; 367: 73-84

7 Zuo XL, Wu P, Ji AM. Nylon filament coated with paraffin for intraluminal permanent middle cerebral artery occlusion in rats. Neuroscience letters 2012; 519: 42-6

8 Toldi J, Rojik I, Feher O. Neonatal monocular enucleation-induced cross-modal effects observed in the cortex of adult rat. Neuroscience 1994; 62: 105-14

9 Farkas T, Perge J, Kis Z, Wolff JR, Toldi J. Facial nerve injury-induced disinhibition in the primary motor cortices of both hemispheres. The European journal of neuroscience 2000; 12 : $2190-4$

10 Carmichael ST. Rodent models of focal stroke: size, mechanism, and purpose. NeuroRx : the journal of the American Society for Experimental NeuroTherapeutics 2005; 2: 396-409

11 Morancho A, Garcia-Bonilla L, Barcelo V, Giralt D, Campos-Martorell M, Garcia S, Montaner J, Rosell A. A new method for focal transient cerebral ischaemia by distal compression of the middle cerebral artery. Neuropathology and applied neurobiology 2012; 38: $617-27$

12 Pagnussat AS, Faccioni-Heuser MC, Netto CA, Achaval M. An ultrastructural study of cell death in the CA1 pyramidal field of the hippocapmus in rats submitted to transient global ischemia followed by reperfusion. Journal of anatomy 2007; 211: 589-99 
13 Sugawara T, Fujimura M, Noshita N, Kim GW, Saito A, Hayashi T, Narasimhan P, Maier $\mathrm{CM}$, Chan PH. Neuronal death/survival signaling pathways in cerebral ischemia. NeuroRx : the journal of the American Society for Experimental NeuroTherapeutics 2004; 1: 17-25

14 Schmued LC, Stowers CC, Scallet AC, Xu L. Fluoro-Jade C results in ultra high resolution and contrast labeling of degenerating neurons. Brain research 2005; 1035: 24-31

15 Chen Y, Swanson RA. Astrocytes and brain injury. Journal of cerebral blood flow and metabolism : official journal of the International Society of Cerebral Blood Flow and Metabolism 2003; 23: 137-49

16 Ridet JL, Malhotra SK, Privat A, Gage FH. Reactive astrocytes: cellular and molecular cues to biological function. Trends in neurosciences 1997; 20: 570-7

17 Rother J, Schellinger PD, Gass A, Siebler M, Villringer A, Fiebach JB, Fiehler J, Jansen O, Kucinski T, Schoder V, Szabo K, Junge-Hulsing GJ, Hennerici M, Zeumer H, Sartor K, Weiller C, Hacke W, Kompetenznetzwerk Schlaganfall Study G. Effect of intravenous thrombolysis on MRI parameters and functional outcome in acute stroke <6 hours. Stroke; a journal of cerebral circulation 2002; 33: 2438-45

18 Zanette EM, Roberti C, Mancini G, Pozzilli C, Bragoni M, Toni D. Spontaneous middle cerebral artery reperfusion in ischemic stroke. A follow-up study with transcranial Doppler. Stroke; a journal of cerebral circulation 1995; 26: 430-3 


\section{Legends to Figures}

Fig. 1. The experimental design to induce short-lasting ischaemic periods. The middle cerebral artery was exposed at its trunk (where its diameter was $300 \mu \mathrm{m}$ ), and lifted up by $1200 \mu \mathrm{m}$. Somatosensory evoked responses (SERs) were recorded at the punctum maximum.

Fig. 2. Changes in SERs as a consequence of repeated short-lasting (15-min) ischaemic periods. The amplitudes of the SERs were measured from peak to peak (see insert). The maximum amplitudes in both reperfusion periods were significantly lower than that in the control period (see labelled data range). $\mathrm{N}=8$, means \pm S.E., $* * * \mathrm{p}<0.001$.

Fig. 3. TTC and FJC labelling after a 1-day survival period ( 2 x 15-min MCAO).

TTC labelling of coronal sections of the brain (sections were made from bregma 1, 3 and 4.5). No tissue damage could be observed (A). Fluoro Jade C (FJC) labelling of the contralateral (B) and the ipsilateral (C) sides following left MCA occlusion for $2 \times 15 \mathrm{~min}$. The insert shows the FJC-positive cells at higher magnification.

Fig. 4. FJC, S100 staining and CD11b immunostaining after a 5-day survival period (30-min MCAO). The inserts show the labelled cells at higher magnification. No FJC labelling emerged contralaterally (A). Pronounced FJC staining emerged in the cortex ipsilateral to the MCAO (B). No staining (meaning that the astrocytes were normal) was observed in the contralateral hemisphere (C). S100-positive astrocytes were observed in the ipsilateral hemisphere (D). No labelling of microglia was observed in the contralateral hemisphere (E). An activated phenotype of microglia was observed in the ipsilateral cortex $(\mathrm{F})$. 



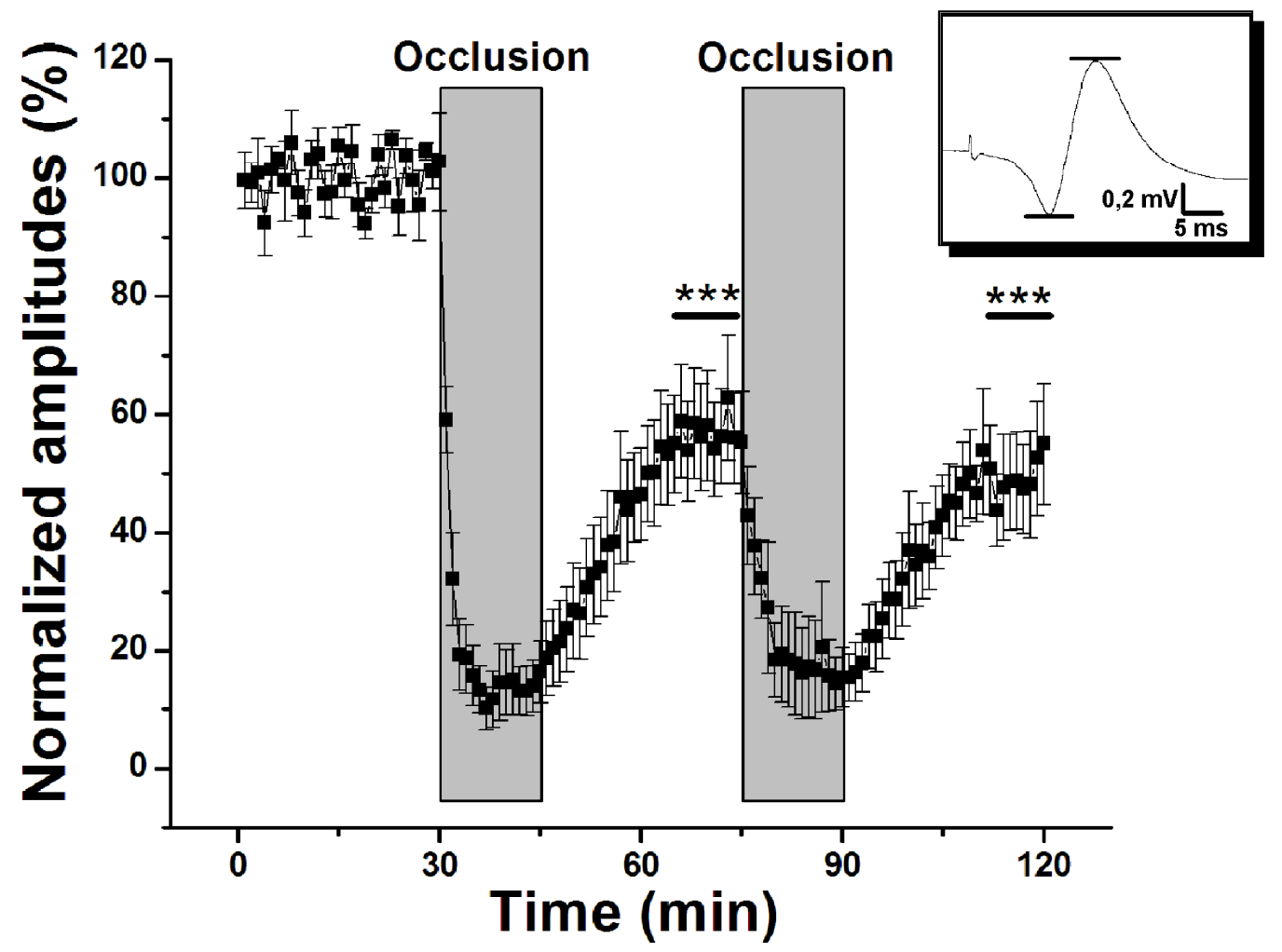

Fig 2 


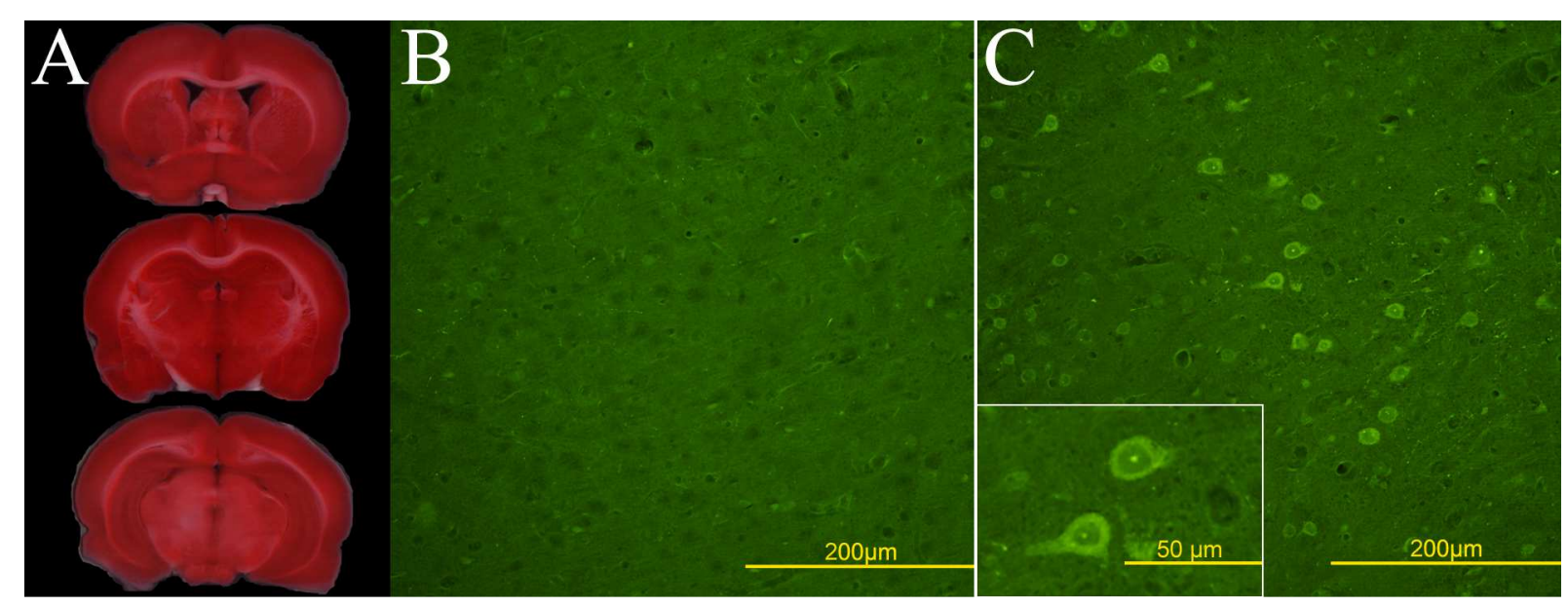

Fig 3 


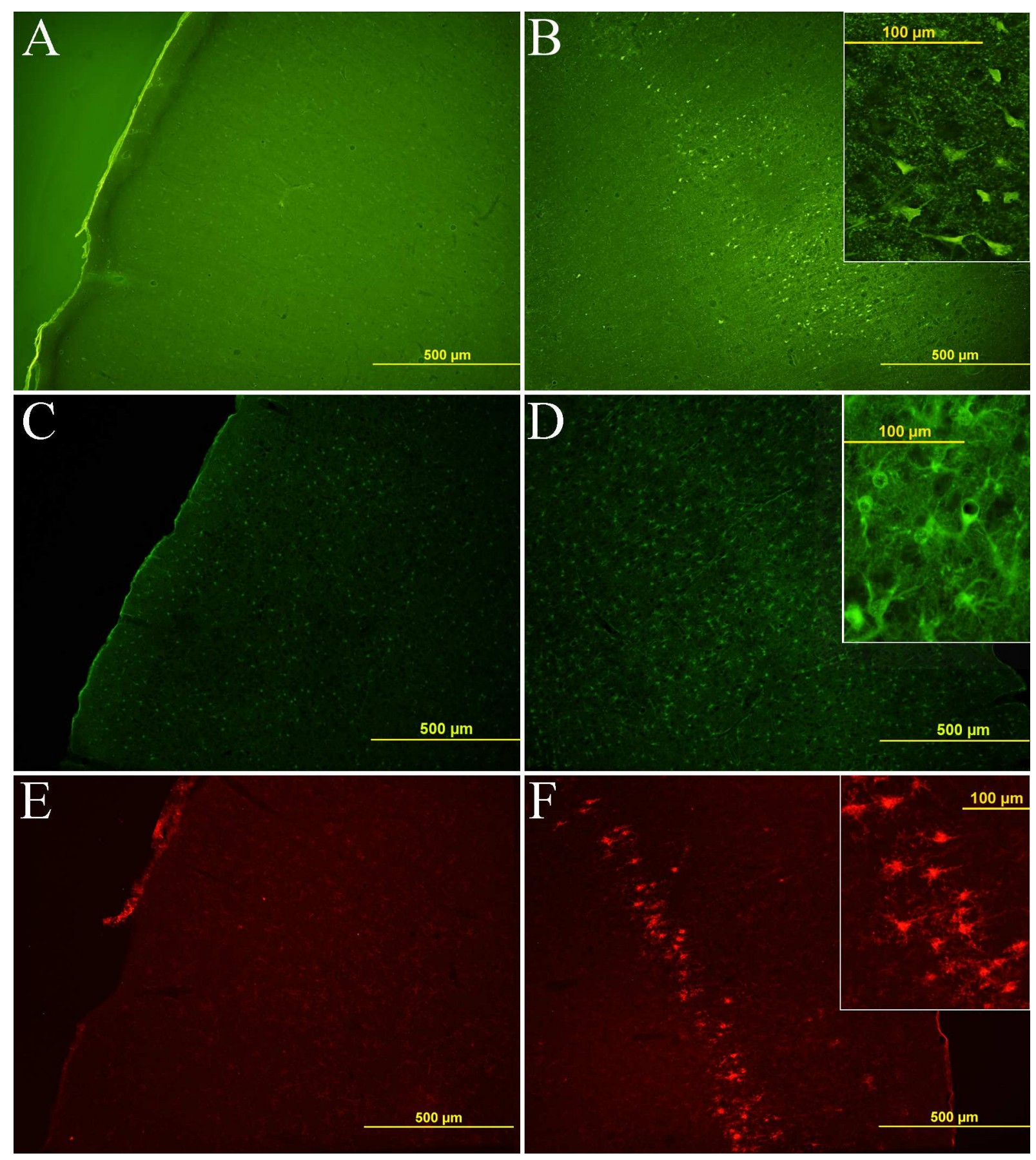

Fig 4 